\title{
Existence and Uniqueness of Periodic Solutions for a Class of Nonlinear Equations with $p$-Laplacian-Like Operators
}

\author{
Hui-Sheng Ding, Guo-Rong Ye, and Wei Long \\ College of Mathematics and Information Science, Jiangxi Normal University, Nanchang, \\ Jiangxi 330022, China \\ Correspondence should be addressed to Wei Long, hopelw@126.com \\ Received 1 February 2010; Accepted 19 March 2010 \\ Academic Editor: Gaston Mandata N'Guerekata \\ Copyright (C) 2010 Hui-Sheng Ding et al. This is an open access article distributed under the \\ Creative Commons Attribution License, which permits unrestricted use, distribution, and \\ reproduction in any medium, provided the original work is properly cited. \\ We investigate the following nonlinear equations with $p$-Laplacian-like operators $\left(\varphi\left(x^{\prime}(t)\right)\right)^{\prime}+$ \\ $f(x(t)) x^{\prime}(t)+g(x(t))=e(t)$ : some criteria to guarantee the existence and uniqueness of periodic \\ solutions of the above equation are given by using Mawhin's continuation theorem. Our results \\ are new and extend some recent results due to Liu (B. Liu, Existence and uniqueness of periodic \\ solutions for a kind of Lienard type $p$-Laplacian equation, Nonlinear Analysis TMA, 69, 724-729, \\ 2008).
}

\section{Introduction}

In this paper, we deal with the existence and uniqueness of periodic solutions for the following nonlinear equations with $p$-Laplacian-like operators:

$$
\left(\varphi\left(x^{\prime}(t)\right)\right)^{\prime}+f(x(t)) x^{\prime}(t)+g(x(t))=e(t)
$$

where $f, g$ are continuous functions on $\mathbb{R}$, and $e$ is a continuous function on $\mathbb{R}$ with period $T>0$; moreover, $\varphi: \mathbb{R} \rightarrow \mathbb{R}$ is a continuous function satisfying the following:

(H1) for any $x_{1}, x_{2} \in \mathbb{R}, x_{1} \neq x_{2},\left[\varphi\left(x_{1}\right)-\varphi\left(x_{2}\right)\right] \cdot\left(x_{1}-x_{2}\right)>0$ and $\varphi(0)=0$;

$(\mathrm{H} 2)$ there exists a function $\alpha:[0,+\infty) \rightarrow[0,+\infty)$ such that $\lim _{s \rightarrow+\infty} \alpha(s)=+\infty$ and

$$
\varphi(x) \cdot x \geq \alpha(|x|)|x|, \quad \forall x \in \mathbb{R} .
$$


It is obvious that under these two conditions, $\varphi$ is an homeomorphism from $\mathbb{R}$ onto $\mathbb{R}$ and is increasing on $\mathbb{R}$.

Recall that $p$-Laplacian equations have been of great interest for many mathematicians. Especially, there is a large literature (see, e.g., [1-7] and references therein) about the existence of periodic solutions to the following $p$-Laplacian equation:

$$
\left(\varphi_{p}\left(x^{\prime}(t)\right)\right)^{\prime}+f(x(t)) x^{\prime}(t)+g(x(t))=e(t)
$$

and its variants, where $\varphi_{p}(s)=|s|^{p-2} s$ for $s \neq 0$ and $\varphi_{p}(0)=0$. Obviously, (1.3) is a special case of (1.1).

However, there are seldom results about the existence of periodic solutions to (1.1). The main difficulty lies in the $p$-Laplacian-like operator $\varphi$ of (1.1), which is more complicated than $\varphi_{p}$ in (1.3). Since there is no concrete form for the $p$-Laplacian-like operator $\varphi$ of (1.1), it is more difficult to prove the existence of periodic solutions to (1.1).

Therefore, in this paper, we will devote ourselves to investigate the existence of periodic solutions to (1.1). As one will see, our theorem generalizes some recent results even for the case of $\varphi(s)=\varphi_{p}(s)$ (see Remark 2.2).

Next, let us recall some notations and basic results. For convenience, we denote

$$
C_{T}^{1}:=\left\{x \in C^{1}(\mathbb{R}, \mathbb{R}): x \text { is } T \text {-periodic }\right\},
$$

which is a Banach space endowed with the norm $\|x\|=\max \left\{|x|_{\infty},\left|x^{\prime}\right|_{\infty}\right\}$, where

$$
|x|_{\infty}=\max _{t \in[0, T]}|x(t)|, \quad\left|x^{\prime}\right|_{\infty}=\max _{t \in[0, T]}\left|x^{\prime}(t)\right| .
$$

In the proof of our main results, we will need the following classical Mawhin's continuation theorem.

Lemma 1.1 ([8]). Let (H1), (H2) hold and $\tilde{f}$ is Carathéodory. Assume that $\Omega$ is an open bounded set in $C_{T}^{1}$ such that the following conditions hold.

(S1) For each $\lambda \in(0,1)$, the problem

$$
\left(\varphi\left(x^{\prime}(t)\right)\right)^{\prime}=\lambda \tilde{f}\left(t, x, x^{\prime}\right), \quad x(0)=x(T), \quad x^{\prime}(0)=x^{\prime}(T)
$$

has no solution on $\partial \Omega$.

(S2) The equation

$$
F(a):=\frac{1}{T} \int_{0}^{T} \tilde{f}(t, a, 0) d t=0
$$

has no solution on $\partial \Omega \cap \mathbb{R}$.

(S3) The Brouwer degree

$$
\operatorname{deg}(F, \Omega \cap \mathbb{R}, 0) \neq 0 .
$$


Then the periodic boundary value problem

$$
\left(\varphi\left(x^{\prime}(t)\right)\right)^{\prime}=\tilde{f}\left(t, x, x^{\prime}\right), \quad x(0)=x(T), \quad x^{\prime}(0)=x^{\prime}(T)
$$

has at least one T-periodic solution on $\bar{\Omega}$.

\section{Main Results}

In this section, we prove an existence and uniqueness theorem for (1.1).

Theorem 2.1. Suppose the following assumptions hold:

(A1) $g \in C^{1}(\mathbb{R}, \mathbb{R})$ and $g^{\prime}(x)<0$ for all $x \in \mathbb{R}$;

(A2) there exist a constant $r \geq 0$ and a function $\varepsilon(t) \in C(\mathbb{R}, \mathbb{R})$ such that for all $t \in \mathbb{R}$ and $|x|>r$,

$$
\begin{gathered}
x[g(x)-\varepsilon(t)]<0, \\
\int_{0}^{T}[\varepsilon(t)-e(t)] d t \leq 0, \quad \int_{0}^{T}|\varepsilon(t)-e(t)| d t<2 .
\end{gathered}
$$

Then (1.1) has a unique T-periodic solution.

Proof

Existence. For the proof of existence, we use Lemma 1.1. First, let us consider the homotopic equation of (1.1):

$$
\left(\varphi\left(x^{\prime}(t)\right)\right)^{\prime}+\lambda f(x(t)) x^{\prime}(t)+\lambda g(x(t))=\lambda e(t), \quad \lambda \in(0,1) .
$$

Let $x(t) \in C_{T}^{1}$ be an arbitrary solution of (2.2). By integrating the two sides of (2.2) over $[0, T]$, and noticing that $x^{\prime}(0)=x^{\prime}(T)$ and $x(0)=x(T)$, we have

$$
\int_{0}^{T}[g(x(t))-e(t)] d t=0
$$

that is,

$$
\frac{1}{T} \int_{0}^{T} g(x(t)) d t=\bar{e}:=\frac{1}{T} \int_{0}^{T} e(t) d t
$$

Since $g(x(\cdot))$ is continuous, there exists $t_{0} \in[0, T]$ such that

$$
g\left(x\left(t_{0}\right)\right) \geq \bar{e} .
$$


In view of (A1), we obtain

$$
x\left(t_{0}\right) \leq \tilde{e},
$$

where $\tilde{e}=g^{-1}(\bar{e})$. Then, for each $t \in\left[t_{0}, t_{0}+T\right]$, we have

$$
\begin{aligned}
2 x(t) & =x(t)+x(t-T) \\
& =x\left(t_{0}\right)+\int_{t_{0}}^{t} x^{\prime}(s) d s+x\left(t_{0}\right)-\int_{t-T}^{t_{0}} x^{\prime}(s) d s \\
& \leq 2 x\left(t_{0}\right)+\int_{t_{0}}^{t}\left|x^{\prime}(s)\right| d s+\int_{t-T}^{t_{0}}\left|x^{\prime}(s)\right| d s \\
& \leq 2 \widetilde{e}+\int_{0}^{T}\left|x^{\prime}(s)\right| d s,
\end{aligned}
$$

which gives that

$$
|x|_{\infty} \leq \tilde{e}+\frac{1}{2} \int_{0}^{T}\left|x^{\prime}(s)\right| d s .
$$

Thus,

$$
|x|_{\infty} \leq|\tilde{e}|+\frac{1}{2} \int_{0}^{T}\left|x^{\prime}(s)\right| d s
$$

Since $\lim _{s \rightarrow+\infty} \alpha(s)=+\infty$, there is a constant $M>0$ such that

$$
\alpha(s) \geq 1, \quad \forall s \geq M
$$

Set

$$
\begin{array}{cc}
E_{1}=\left\{t: t \in[0, T],\left|x^{\prime}(t)\right|>M\right\}, & E_{2}=\left\{t: t \in[0, T],\left|x^{\prime}(t)\right| \leq M\right\}, \\
F_{1}=\{t: t \in[0, T],|x(t)|>r\}, & F_{2}=\{t: t \in[0, T],|x(t)| \leq r\} .
\end{array}
$$

In view of (A2) and

$$
\varphi(x) \cdot x \geq \alpha(|x|)|x|, \quad \forall x \in \mathbb{R},
$$


we get

$$
\begin{aligned}
& \int_{0}^{T}\left|x^{\prime}(t)\right| d t=\int_{E_{1}}\left|x^{\prime}(t)\right| d t+\int_{E_{2}}\left|x^{\prime}(t)\right| d t \\
& \leq \int_{E_{1}}\left|x^{\prime}(t)\right| d t+M T \\
& \leq \int_{E_{1}} \frac{\varphi\left(x^{\prime}(t)\right) x^{\prime}(t)}{\alpha\left(\left|x^{\prime}(t)\right|\right)} d t+M T \\
& \leq \int_{E_{1}} \varphi\left(x^{\prime}(t)\right) x^{\prime}(t) d t+M T \\
& \leq \int_{0}^{T} \varphi\left(x^{\prime}(t)\right) x^{\prime}(t) d t+M T \\
& =\int_{0}^{T} \varphi\left(x^{\prime}(t)\right) d x(t)+M T \\
& =-\int_{0}^{T}\left(\varphi\left(x^{\prime}(t)\right)\right)^{\prime} x(t) d t+M T \\
& =\lambda \int_{0}^{T}[g(x(t))-e(t)] x(t) d t+\lambda \int_{0}^{T} f(x(t)) x^{\prime}(t) x(t) d t+M T \\
& =\lambda \int_{0}^{T}[g(x(t))-e(t)] x(t) d t+M T \\
& =\lambda \int_{F_{1}}[g(x(t))-\varepsilon(t)] x(t) d t+\lambda \int_{F_{2}}[g(x(t))-\varepsilon(t)] x(t) d t \\
& +\lambda \int_{0}^{T}[\varepsilon(t)-e(t)] x(t) d t+M T \\
& \leq \int_{F_{2}}|g(x(t))-\varepsilon(t)| \cdot|x(t)| d t+M T+\int_{0}^{T}|\varepsilon(t)-e(t)| d t \cdot|x|_{\infty} \\
& \leq M^{\prime} T+M T+\int_{0}^{T}|\varepsilon(t)-e(t)| d t \cdot|x|_{\infty}
\end{aligned}
$$

where

$$
M^{\prime}=\left[\max _{|x| \leq r}|g(x)|+\max _{t \in[0, T]}|\varepsilon(t)|\right] \cdot r .
$$

By (2.9), we have

$$
|x|_{\infty} \leq|\tilde{e}|+\frac{\left(M^{\prime}+M\right) T}{2}+\frac{1}{2} \int_{0}^{T}|\varepsilon(t)-e(t)| d t \cdot|x|_{\infty} .
$$


Noticing that

$$
\int_{0}^{T}|\varepsilon(t)-e(t)| d t<2
$$

there exists a constant $M^{\prime \prime}>|\tilde{e}|$ such that

$$
|x|_{\infty} \leq M^{\prime \prime}
$$

On the other hand, it follows from

$$
|\varphi(x)| \cdot|x|=\varphi(x) \cdot x \geq \alpha(|x|)|x|, \quad \forall x \in \mathbb{R}
$$

that $\alpha(|x|) \leq|\varphi(x)|$ for $x \neq 0$. In addition, since $x(0)=x(T)$, there exists $t_{1} \in[0, T]$ such that $x^{\prime}\left(t_{1}\right)=0$. Thus $\varphi\left(x^{\prime}\left(t_{1}\right)\right)=0$.

Then, for all $t \in E:=\left\{t \in[0, T]: x^{\prime}(t) \neq 0\right\}$, we have

$$
\begin{aligned}
\alpha\left(\left|x^{\prime}(t)\right|\right) & \leq\left|\varphi\left(x^{\prime}(t)\right)\right| \\
& =\left|\int_{t_{1}}^{t}\left(\varphi\left(x^{\prime}(s)\right)\right)^{\prime} d s\right| \\
& \leq\left|\int_{t_{1}}^{t} f(x(s)) \cdot x^{\prime}(s) d s\right|+\int_{0}^{T}|g(x(s))| d s+\int_{0}^{T}|e(s)| d s \\
& \leq\left|\int_{x\left(t_{1}\right)}^{x(t)} f(u) d u\right|+\left[\max _{|x| \leq M^{\prime \prime}}|g(x)|+\max _{t \in[0, T]}|e(t)|\right] \cdot T \\
& \leq \int_{-M^{\prime \prime}}^{M^{\prime \prime}}|f(u)| d u+\left[\max _{|x| \leq M^{\prime \prime}}|g(x)|+\max _{t \in[0, T]}|e(t)|\right] \cdot T \\
& \leq \max _{|x| \leq M^{\prime \prime}}|f(x)| \cdot 2 M^{\prime \prime}+\left[\max _{|x| \leq M^{\prime \prime}}|g(x)|+\max _{t \in[0, T]}|e(t)|\right] \cdot T:=M^{\prime \prime \prime} .
\end{aligned}
$$

For the above $M^{\prime \prime \prime}$, it follows from $\lim _{s \rightarrow+\infty} \alpha(s)=+\infty$ that there exists $G>M^{\prime \prime}$ such that

$$
\alpha(s)>M^{\prime \prime \prime}, \quad s \geq G
$$

Combining this with $\alpha\left(\left|x^{\prime}(t)\right|\right) \leq M^{\prime \prime \prime}$, we get

$$
\left|x^{\prime}(t)\right|<G, \quad t \in E,
$$

which yields that $\left|x^{\prime}\right|_{\infty}<G$.

Now, we have proved that any solution $x(t) \in C_{T}^{1}$ of (2.2) satisfies

$$
|x|_{\infty}<G, \quad\left|x^{\prime}\right|_{\infty}<G
$$


Since $G>|\tilde{e}|$, we have

$$
G>\widetilde{e}=g^{-1}(\bar{e}), \quad-G<\tilde{e}=g^{-1}(\bar{e})
$$

In view of $g$ being strictly decreasing, we get

$$
g(G)<\bar{e}, \quad g(-G)>\bar{e}
$$

Set

$$
\Omega=\left\{x \in C_{T}^{1}:|x|_{\infty} \leq G,\left|x^{\prime}\right|_{\infty} \leq G\right\} .
$$

Then, we know that (2.2) has no solution on $\partial \Omega$ for each $\lambda \in(0,1)$, that is, the assumption (S1) of Lemma 1.1 holds. In addition, it follows from (2.24) that

$$
\begin{aligned}
-\frac{1}{T} \int_{0}^{T}[g(G)-e(t)] d t & =\bar{e}-g(G)>0 \\
-\frac{1}{T} \int_{0}^{T}[g(-G)-e(t)] d t & =\bar{e}-g(-G)<0 .
\end{aligned}
$$

So the assumption (S2) of Lemma 1.1 holds. Let

$$
H(x, \mu)=\mu x-(1-\mu) \frac{1}{T} \int_{0}^{T}[g(x)-e(t)] d t .
$$

For $x \in \partial \Omega \cap \mathbb{R}$ and $\mu \in[0,1]$, by (2.24), we have

$$
\begin{aligned}
x H(x, \mu) & =\mu x^{2}-(1-\mu) x \frac{1}{T} \int_{0}^{T}[g(x)-e(t)] d t \\
& =\mu x^{2}+(1-\mu) x \frac{1}{T} \int_{0}^{T}[\bar{e}-g(x)] d t>0 .
\end{aligned}
$$

Thus, $H(x, \mu)$ is a homotopic transformation. So

$$
\begin{aligned}
\operatorname{deg}(F, \Omega \cap \mathbb{R}, 0) & =\operatorname{deg}(H(x, 0), \Omega \cap \mathbb{R}, 0) \\
& =\operatorname{deg}(H(x, 1), \Omega \cap \mathbb{R}, 0) \\
& =\operatorname{deg}(I, \Omega \cap \mathbb{R}, 0) \neq 0,
\end{aligned}
$$

that is, the assumption (S3) of Lemma 1.1 holds. By applying Lemma 1.1, there exists at least one solution with period $T$ to (1.1). 
Uniqueness. Let

$$
\psi(x)=\int_{0}^{x} f(u) d u, \quad y(t)=\varphi\left(x^{\prime}(t)\right)+\psi(x(t)) .
$$

Then (1.1) is transformed into

$$
\begin{aligned}
& x^{\prime}(t)=\varphi^{-1}[y(t)-\psi(x(t))], \\
& y^{\prime}(t)=-g(x(t))+e(t) .
\end{aligned}
$$

Let $x_{1}(t)$ and $x_{2}(t)$ being two $T$-periodic solutions of (1.1); and

$$
y_{i}(t)=\varphi\left(x_{i}^{\prime}(t)\right)+\psi\left(x_{i}(t)\right), \quad i=1,2 .
$$

Then we obtain

$$
\begin{aligned}
& x_{i}^{\prime}(t)=\varphi^{-1}\left[y_{i}(t)-\psi\left(x_{i}(t)\right)\right], \\
& y_{i}^{\prime}(t)=-g\left(x_{i}(t)\right)+e(t) .
\end{aligned} \quad i=1,2
$$

Setting

$$
v(t)=x_{1}(t)-x_{2}(t), \quad u(t)=y_{1}(t)-y_{2}(t),
$$

it follows from (2.33) that

$$
\begin{aligned}
& v^{\prime}(t)=\varphi^{-1}\left[y_{1}(t)-\psi\left(x_{1}(t)\right)\right]-\varphi^{-1}\left[y_{2}(t)-\psi\left(x_{2}(t)\right)\right], \\
& u^{\prime}(t)=-\left[g\left(x_{1}(t)\right)-g\left(x_{2}(t)\right)\right] .
\end{aligned}
$$

Now, we claim that

$$
u(t) \leq 0, \quad \forall t \in \mathbb{R} .
$$

If this is not true, we consider the following two cases.

Case 1. There exists $t_{2} \in(0, T)$ such that

$$
u\left(t_{2}\right)=\max _{t \in[0, T]} u(t)=\max _{t \in \mathbb{R}} u(t)>0,
$$

which implies that

$$
\begin{gathered}
u^{\prime}\left(t_{2}\right)=-\left[g\left(x_{1}\left(t_{2}\right)\right)-g\left(x_{2}\left(t_{2}\right)\right)\right]=0, \\
u^{\prime \prime}\left(t_{2}\right)=-\left.\left[g\left(x_{1}(t)\right)-g\left(x_{2}(t)\right)\right]^{\prime}\right|_{t=t_{2}}=-\left[g^{\prime}\left(x_{1}\left(t_{2}\right)\right) x_{1}^{\prime}\left(t_{2}\right)-g^{\prime}\left(x_{2}\left(t_{2}\right)\right) x_{2}^{\prime}\left(t_{2}\right)\right] \leq 0 .
\end{gathered}
$$


By (A1), $g^{\prime}(x)<0$. So it follows from $g\left(x_{1}\left(t_{2}\right)\right)-g\left(x_{2}\left(t_{2}\right)\right)=0$ that $x_{1}\left(t_{2}\right)=x_{2}\left(t_{2}\right)$. Thus, in view of

$$
-g^{\prime}\left(x_{1}\left(t_{2}\right)\right)>0, \quad u\left(t_{2}\right)=y_{1}\left(t_{2}\right)-y_{2}\left(t_{2}\right)>0,
$$

and (H1), we obtain

$$
\begin{aligned}
u^{\prime \prime}\left(t_{2}\right) & =-g^{\prime}\left(x_{1}\left(t_{2}\right)\right)\left[x^{\prime}\left(t_{2}\right)-x_{2}^{\prime}\left(t_{2}\right)\right] \\
& =-g^{\prime}\left(x_{1}\left(t_{2}\right)\right)\left\{\varphi^{-1}\left[y_{1}\left(t_{2}\right)-\psi\left(x_{1}\left(t_{2}\right)\right)\right]-\varphi^{-1}\left[y_{2}\left(t_{2}\right)-\psi\left(x_{2}\left(t_{2}\right)\right)\right]\right\} \\
& =-g^{\prime}\left(x_{1}\left(t_{2}\right)\right)\left\{\varphi^{-1}\left[y_{1}\left(t_{2}\right)-\psi\left(x_{1}\left(t_{2}\right)\right)\right]-\varphi^{-1}\left[y_{2}\left(t_{2}\right)-\psi\left(x_{1}\left(t_{2}\right)\right)\right]\right\}>0,
\end{aligned}
$$

which contradicts with $u^{\prime \prime}\left(t_{2}\right) \leq 0$.

Case 2.

$$
u(0)=\max _{t \in[0, T]} u(t)=\max _{t \in \mathbb{R}} u(t)>0 .
$$

Also, we have $u^{\prime}(0)=0$ and $u^{\prime \prime}(0) \leq 0$. Then, similar to the proof of Case 1 , one can get a contradiction.

Now, we have proved that

$$
u(t) \leq 0, \quad \forall t \in \mathbb{R} .
$$

Analogously, one can show that

$$
u(t) \geq 0, \quad \forall t \in \mathbb{R} .
$$

So we have $u(t) \equiv 0$. Then, it follows from (2.35) that

$$
g\left(x_{1}(t)\right)-g\left(x_{2}(t)\right) \equiv 0, \quad \forall t \in \mathbb{R},
$$

which implies that

$$
x_{2}(t) \equiv x_{1}(t), \quad \forall t \in \mathbb{R}
$$

Hence, (1.1) has a unique T-periodic solution. The proof of Theorem 2.1 is now completed.

Remark 2.2. In Theorem 2.1, setting $\varepsilon(t) \equiv e(t)$, then (A2) becomes as follows:

(A2 ${ }^{\prime}$ ) there exists a constant $r \geq 0$ such that for all $t \in \mathbb{R}$ and $|x|>r$,

$$
x[g(x)-e(t)]<0 .
$$


In the case $\varphi(s)=\varphi_{p}(s)$, Liu [7, Theorem 1] proved that (1.1) has a unique $T$-periodic solution under the assumptions (A1) and $\left(\mathrm{A} 2^{\prime}\right)$. Thus, even for the case of $\varphi(s)=\varphi_{p}(s)$, Theorem 2.1 is a generalization of $[7$, Theorem 1$]$.

In addition, we have the following interesting corollary.

Corollary 2.3. Suppose (A1) and

$\left(A 2^{\prime \prime}\right)$ there exist a constant $\alpha \geq 0$ such that

$$
\int_{0}^{T}[g(\alpha)-e(t)] d t \leq 0, \quad \int_{0}^{T}|g(\alpha)-e(t)| d t<2
$$

hold. Then (1.1) has a unique T-periodic solution.

Proof. Let $\varepsilon(t) \equiv g(\alpha)$. Noticing that

$$
x[g(x)-g(\alpha)]<0, \quad|x|>\alpha,
$$

we know that (A2) holds with $r=\alpha$. This completes the proof.

At last, we give two examples to illustrate our results.

Example 2.4. Consider the following nonlinear equation:

$$
\left(\varphi\left(x^{\prime}(t)\right)\right)^{\prime}+f(x(t)) x^{\prime}(t)+g(x(t))=e(t)
$$

where $\varphi(x)=2 x e^{x^{2}}-2 x, f(x)=e^{-x}, g(x)=-\left(x^{3}+x\right)$, and $e(t)=\sin t$. One can easily check that $\varphi$ satisfy (H1) and (H2). Obviously, (A1) holds. Moreover, since

$$
\lim _{x \rightarrow+\infty} g(x)=-\infty, \quad \lim _{x \rightarrow-\infty} g(x)=+\infty
$$

it is easy to verify that (A2) holds. By Theorem 2.1, (2.49) has a unique $2 \pi$-periodic solution. Example 2.5. Consider the following $p$-Laplacian equation:

$$
\left(\varphi_{p}\left(x^{\prime}(t)\right)\right)^{\prime}+g(x(t))=e(t)
$$

where $g(x)=-(1 / 2) \arctan x$, and $e(t)=\sin ^{2} t$. Obviously, (A1) holds. Moreover, we have

$$
\int_{0}^{\pi}[g(0)-e(t)] d t \leq 0, \quad \int_{0}^{\pi}|g(0)-e(t)| d t=\int_{0}^{\pi} \sin ^{2} t=\frac{\pi}{2}<2 .
$$

So (A2") holds. Then, by Corollary 2.3, (2.51) has a unique $\pi$-periodic solution. 
Remark 2.6. In Example 2.5, $\forall r>0$, we have

$$
x\left(g(x)-e\left(\frac{\pi}{2}\right)\right)>r\left(1-\frac{\pi}{4}\right)>0, \quad \forall x<-r .
$$

Thus, (A2') does not hold. So [7, Theorem 1] cannot be applied to Example 2.5. This means that our results generalize [7, Theorem 1] in essence even for the case of $\varphi(s)=\varphi_{p}(s)$.

\section{Acknowledgments}

The authors are grateful to the referee for valuable suggestions and comments, which improved the quality of this paper. The work was supported by the NSF of China, the NSF of Jiangxi Province of China (2008GQS0057), the Youth Foundation of Jiangxi Provincial Education Department (GJJ09456), and the Youth Foundation of Jiangxi Normal University.

\section{References}

[1] S. Lu, "New results on the existence of periodic solutions to a $p$-Laplacian differential equation with a deviating argument," Journal of Mathematical Analysis and Applications, vol. 336, no. 2, pp. 1107-1123, 2007.

[2] S. Lu, "Existence of periodic solutions to a $p$-Laplacian Liénard differential equation with a deviating argument," Nonlinear Analysis: Theory, Methods E Applications, vol. 68, no. 6, pp. 1453-1461, 2008.

[3] B. Liu, "Periodic solutions for Liénard type $p$-Laplacian equation with a deviating argument," Journal of Computational and Applied Mathematics, vol. 214, no. 1, pp. 13-18, 2008.

[4] W.-S. Cheung and J. Ren, "Periodic solutions for $p$-Laplacian Liénard equation with a deviating argument," Nonlinear Analysis: Theory, Methods \& Applications, vol. 59, no. 1-2, pp. 107-120, 2004.

[5] W.-S. Cheung and J. Ren, "Periodic solutions for $p$-Laplacian differential equation with multiple deviating arguments," Nonlinear Analysis: Theory, Methods \& Applications, vol. 62, no. 4, pp. 727-742, 2005.

[6] W.-S. Cheung and J. Ren, "Periodic solutions for $p$-Laplacian Rayleigh equations," Nonlinear Analysis: Theory, Methods \& Applications, vol. 65, no. 10, pp. 2003-2012, 2006.

[7] B. Liu, "Existence and uniqueness of periodic solutions for a kind of Lienard type $p$-Laplacian equation," Nonlinear Analysis: Theory, Methods E Applications, vol. 69, no. 2, pp. 724-729, 2008.

[8] R. Manásevich and J. Mawhin, "Periodic solutions for nonlinear systems with $p$-Laplacian-like operators," Journal of Differential Equations, vol. 145, no. 2, pp. 367-393, 1998. 